\title{
Continuous Cardiac Output Monitoring by Peripheral Blood Pressure Waveform Analysis
}

\author{
Ramakrishna Mukkamala*, Member, IEEE, Andrew T. Reisner, Horacio M. Hojman, \\ Roger G. Mark, Senior Member, IEEE, and Richard J. Cohen
}

\begin{abstract}
A clinical method for monitoring cardiac output (CO) should be continuous, minimally invasive, and accurate. However, none of the conventional $\mathrm{CO}$ measurement methods possess all of these characteristics. On the other hand, peripheral arterial blood pressure (ABP) may be measured reliably and continuously with little or no invasiveness. We have developed a novel technique for continuously monitoring changes in $\mathrm{CO}$ by mathematical analysis of a peripheral ABP waveform. In contrast to the previous techniques, our technique analyzes the ABP waveform over time scales greater than a cardiac cycle in which the confounding effects of complex wave reflections are attenuated. The technique specifically analyzes 6-min intervals of ABP to estimate the pure exponential pressure decay that would eventually result if pulsatile activity abruptly ceased (i.e., after the high frequency wave reflections vanish). The technique then determines the time constant of this exponential decay, which equals the product of the total peripheral resistance and the nearly constant arterial compliance, and computes proportional CO via Ohm's law. To validate the technique, we performed six acute swine experiments in which peripheral ABP waveforms and aortic flow probe $\mathrm{CO}$ were simultaneously measured over a wide physiologic range. We report an overall CO error of $14.6 \%$.
\end{abstract}

Index Terms-Arterial blood pressure, arterial tree, cardiac output, distributed effects, hemodynamics, mathematical modeling, pulse contour analysis, system identification, wave reflections, Windkessel.

\section{INTRODUCTION}

A clinical method for monitoring cardiac output (CO) should be continuous, minimally invasive, accurate, and inexpensive. However, none of the conventional CO measurement methods possess all of these characteristics [1]. Consider, for example, the thermodilution method, which is employed in most intensive care units and surgical suites. Although the method is relatively inexpensive, it does not operate continuously, requires a pulmonary artery catheterization whose safety

Manuscript received June 8, 2004; revised June 11, 2005. This work was supported in part by the National Aeronautics and Space Administration (NASA) through the NASA Cooperative Agreement NCC 9-58 with the National Space Biomedical Research Institute and in part by a grant from the Center for the Integration of Medicine and Innovative Technology, and Michigan State University. Asterisk indicates corresponding author.

*R. Mukkamala is with the Department of Electrical and Computer Engineering, Michigan State University, East Lansing, MI 48824 USA (e-mail: rama@egr.msu.edu).

A. T. Reisner is with the Department of Emergency Medicine, Massachusetts General Hospital, Boston, MA 02114 USA.

H. M. Hojman is with the Department of Surgery, Yale University School of Medicine, New Haven, CT 06519 USA.

R. G. Mark and R. J. Cohen are with the Harvard-MIT Division of Health Sciences and Technology, MIT, Cambridge, MA 02139 USA.

Digital Object Identifier 10.1109/TBME.2005.869780 has been questioned (e.g., [2]), and is not very accurate due to the many assumptions upon which it is based [1]. The most accurate method for measuring $\mathrm{CO}$ involves placing an ultrasonic flow probe around the aorta. But, this method requires the drastic procedure of opening the chest.

While the measurement of $\mathrm{CO}$ has proven to be difficult, peripheral arterial blood pressure (ABP), which is related to $\mathrm{CO}$ through the arterial tree, may be measured reliably and continuously with little or no invasiveness via radial artery catheterization, arterial tonometry [3], and finger-cuff photoplethysmography [4]. Indeed, since at least 1904 [5], investigators have proposed analysis techniques to monitor $\mathrm{CO}$ from $\mathrm{ABP}$ waveforms.

Much of the earlier work assumed that the arterial tree is well represented by a two-parameter Windkessel model accounting for the true total compliance of the large arteries [arterial compliance (AC)] and the total peripheral resistance (TPR) of the small arteries (see electrical analog in Fig. 1). While techniques based on this model generally failed when applied to ABP waveforms measured centrally in the aorta (e.g., [6] and [7]), Bourgeois et al. demonstrated that their technique yielded a quantity which varied linearly with aortic flow probe $\mathrm{CO}$ over a wide physiologic range [8]. The basic concept of their technique is that, according to the Windkessel model, ABP should decay like a pure exponential during each diastolic interval with a time constant equal to the product of TPR and AC (Windkessel time constant, $\tau$ ). Since AC is nearly constant over a wide pressure range and on the time scale of months [8]-[11], CO could then be measured to within a constant scale factor by dividing the time-averaged ABP with $\tau$. Thus, the technique of Bourgeois et al. involves fitting an exponential function to the diastolic interval of each ABP pulse in order to measure $\tau$ (see Fig. 1).

Bourgeois et al. were able to validate their technique with respect to central ABP waveforms, because the diastolic interval of these waveforms can resemble an exponential decay following incisura [Fig. 2(a)]. These investigators specifically identified a location in the thoracic aorta a few centimeters cephalad of the dorsal insertion of the diaphragm as the optimal site in the canine arterial tree for observing an exponential diastolic decay. However, central ABP is rarely measured clinically because of the risk of blood clot formation and embolization. Moreover, in readily available peripheral ABP waveforms, an exponential diastolic decay is usually not apparent [Fig. 2(b)]. Indeed, it is well known that the contour of the arterial pulse changes significantly as it traverses through the arterial tree [12]. The reason is that the arterial tree is not simply a lumped system like the Windkessel model suggests but rather a complicated distributed system with impedance 


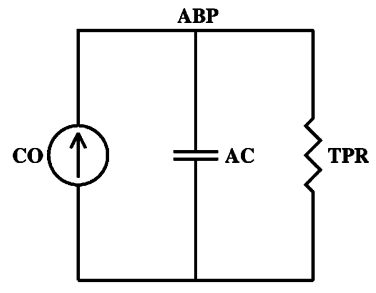

$\tau=\mathrm{TPR} \cdot \mathrm{AC}$
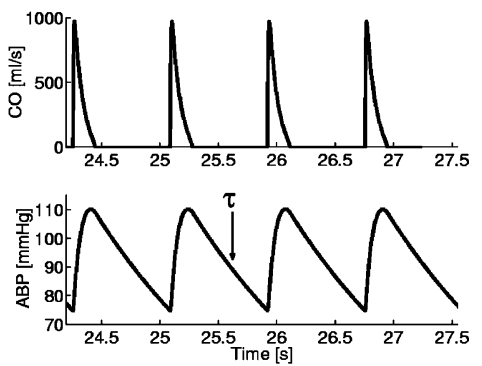

Fig. 1. The technique of Bourgeois et al. for monitoring $\mathrm{CO}$ from an ABP waveform [8]. According to the Windkessel model (left), ABP should decay like a pure exponential during each diastolic interval with a time constant $(\tau)$ equal to the product of the TPR and the nearly constant AC. Thus, their technique involves first fitting an exponential to the diastolic interval of each ABP pulse to determine $\tau$ (right) and then dividing the time-averaged ABP (MAP) with $\tau$ to estimate proportional $\mathrm{CO}$. (a)

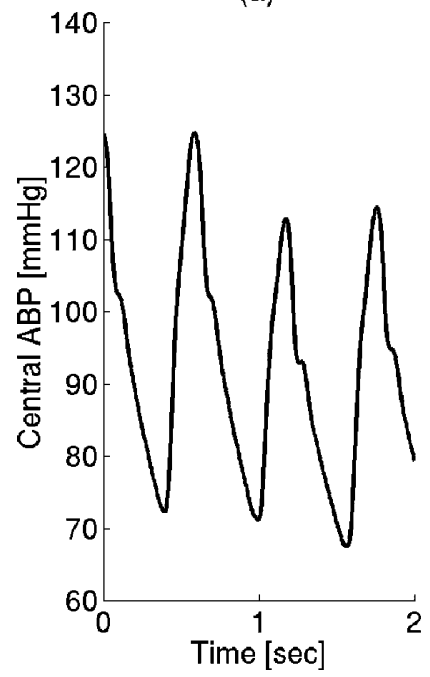

(b)
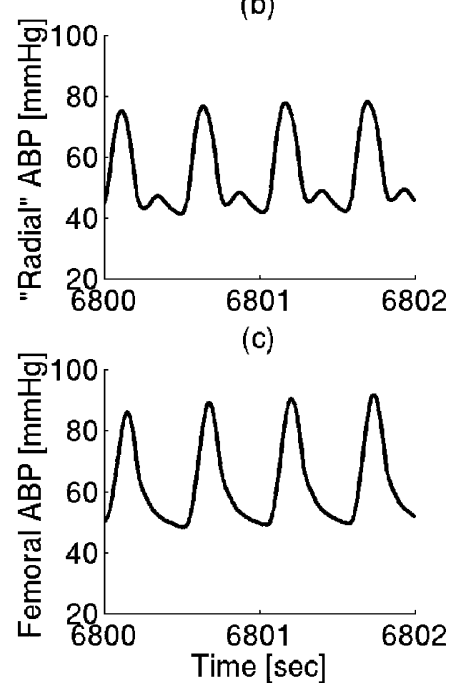

Fig. 2. Example samples of measured (a) central, (b) "radial," and (c) femoral ABP in swine. The diastolic intervals of the central ABP waveform resemble exponential decays; however, central ABP is rarely measured clinically. In contrast, pure exponential diastolic decays are not evident in the "radial" or femoral ABP waveforms, as they are corrupted by complex wave reflections. Thus, the technique of Bourgeois et al. in Fig. 1 cannot be applied to readily measured peripheral ABP waveforms.

mismatches throughout due to vessel tapering, bifurcations, and caliber changes. Thus, the diastolic (and systolic) intervals of peripheral ABP waveforms are corrupted by complex wave reflections that occur at each and every site of impedance mismatch. (Note that the complexity of these sites and their varying distances from the aorta result in reflected waves with large phasic differences, which can tend to mitigate the cumulative effects of these waves on the central ABP waveform, i.e., destructive interference [13].) The technique of Bourgeois et $a l$. , therefore, cannot be applied to readily measured peripheral ABP waveforms. In fact, its application to central ABP waveforms may also be somewhat limited, as Cundick et al. reported that they could not identify such an optimal location in the human aorta in which the diastolic interval of the ABP waveform appeared as a pure exponential decay [14].

More recent techniques have aimed to monitor $\mathrm{CO}$ from ABP waveforms measured peripherally. Techniques based on an adaptive aorta model, which require ABP waveforms measured at two peripheral sites, have been proposed [15], [16]. Learning techniques, which require large training data sets consisting of simultaneous measurements of $\mathrm{CO}$ and $\mathrm{ABP}$ waveforms obtained over the entire physiologic range, have also been suggested [17]-[19]. To our knowledge, only Wesseling et al. [20] and Linton et al. [21] have proposed techniques that are intended to analyze a single peripheral ABP waveform (see Section V).

The common feature of all previous techniques for monitoring $\mathrm{CO}$ from continuous $\mathrm{ABP}$ is that the waveform analysis is employed only over time scales within a cardiac cycle. Because of the corruptive effects of wave reflections at these time scales, these techniques were limited in that they 1) could only be applied to central ABP waveforms in which the cumulative effects of the wave reflections are attenuated; 2) necessitated multiple peripheral $\mathrm{ABP}$ waveform measurements, which are usually not obtained clinically; 3 ) required a large training data set obtained over the entire physiologic range; and/or 4) could potentially be subject to significant error due to the extremely complicated wave phenomena whose nature differs substantially between subjects and disease states as well as at various measurement sites in the arterial tree. Thus, despite a century of effort, a clinical technique for monitoring $\mathrm{CO}$ from $\mathrm{ABP}$ has not been widely adopted.

In this paper, we introduce a fundamentally new mathematical analysis technique for continuously monitoring changes in $\mathrm{CO}$ from a single peripheral $\mathrm{ABP}$ waveform. To demonstrate the validity of the technique, we present results from six acute swine experiments in which peripheral ABP waveforms and ultrasonic aortic flow probe $\mathrm{CO}$ were simultaneously measured during a set of interventions, which altered $\mathrm{CO}$ and other hemodynamic parameters over a wide physiologic range.

\section{The TECHNIQUE}

Our technique is inspired by transmission line theory, which predicts that the confounding effects of wave reflections diminish with increasing time scale. That is, the wave reflections significantly corrupt peripheral ABP waveforms over short time scales (high frequencies) without complicating the waveform over longer time scales (low frequencies) [13]. For example, consider the limiting case in which the time scale is sufficiently long such that the wavelengths of the propagating waves are much larger than the dimension of the arterial tree. At such time scales, the arterial tree acts as a single blood reservoir, and the Windkessel model is therefore valid. So, for example, if pulsatile activity abruptly ceased, then peripheral ABP may eventually decay like a pure exponential as soon as the faster wave reflections died out. Furthermore, the arterial tree is being continuously excited at time scales greater than a cardiac cycle by ongoing perturbations (e.g., breathing) and the compensatory response of the regulatory system (e.g., baroreflex system) [22]. Thus, there is significant long time scale information in ABP waveforms. These important concepts are demonstrated in Fig. 3, which illustrates two ABP waveforms measured at the same time but at different sites in the arterial tree. The short time scale (within a beat) variations are different in the two waveforms, as the characteristics of the highly complex wave 

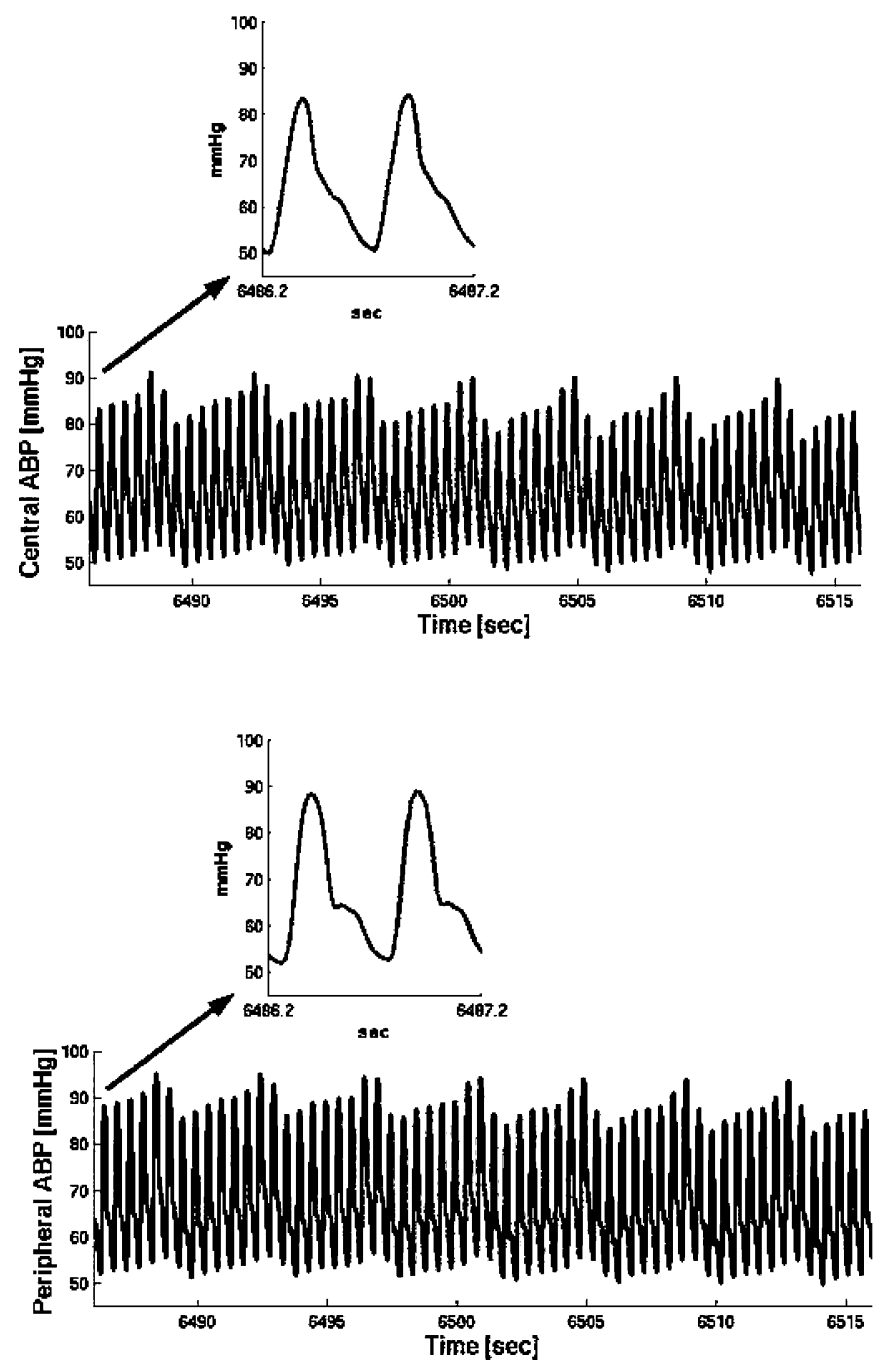

Fig. 3. Two swine ABP waveforms simultaneously measured from the central aorta and the "radial" artery. While the short time scale variations (insets) differ due to highly complex wave effects, the long time scale variations are more similar as the wave phenomena only confound the high frequencies. This implies that if pulsatile activity abruptly ceased, then peripheral ABP would eventually decay like a pure exponential (according to the time constant $\tau$ of the Windkessel model of Fig. 1) once the faster wave effects vanished.

reflections differ at the two measurement sites. In contrast, the long time scale (beat-to-beat) variations are much more similar, since the confounding effects of the wave reflections are less significant over these time scales.

Our technique therefore analyzes the ABP waveform over long time intervals (seconds to minutes) in order to determine the pure exponential decay that would eventually result if pulsatile activity abruptly ceased. More specifically, the ABP response to a single, solitary cardiac contraction is estimated from a digitized ABP waveform $(h(t)$ in Fig. 4). Then, the Windkessel time constant $\tau$ is measured by fitting an exponential to the tail end of this estimated response once the faster wave reflections have vanished (Fig. 4). Finally, proportional CO is computed via Ohm's law. The technique, which is illustrated in Fig. 4, is specifically implemented in four mathematical steps.

First, a cardiac contractions signal is constructed through the formation of an impulse train in which each impulse is located at the onset of upstroke of an ABP pulse and has an area equal

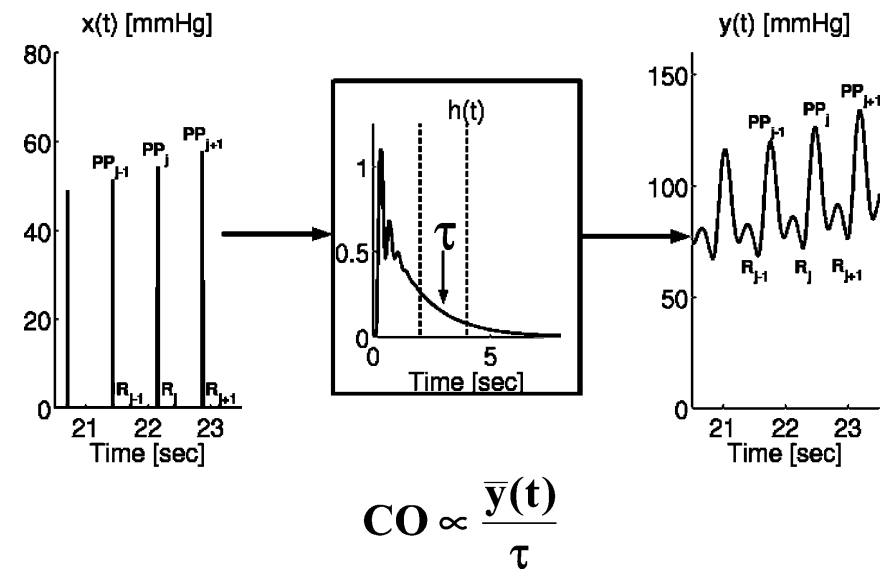

Fig. 4. The technique introduced herein for monitoring $\mathrm{CO}$ from a peripheral ABP waveform. The ABP waveform $(y(t))$ is analyzed over long time intervals (seconds to minutes) so as to estimate the ABP response to a single cardiac contraction $(h(t))$ [see (1) and (2)]. The time constant $\tau$ of the Windkessel model of Fig. 1 is then determined by fitting an exponential to the tail end of $h(t)$ once the faster wave reflections have vanished [see (3)]. Finally, proportional $\mathrm{CO}$ is computed by dividing the time-averaged ABP $(\bar{y}(t))$ with $\tau$ [see (4)]. Note that $\tau$ is accurately determined by analysis of the subtle, beat-to-beat ABP variations in which wave effects are no longer a confounding factor (Fig. 3).

to the ensuing pulse pressure (PP). $\mathrm{PP}$ is determined as the maximum ABP value minus the ABP value at the onset of upstroke.

Then, the relationship between the cardiac contractions signal $(x(t))$ and the ABP waveform $(y(t))$ is characterized by estimating an impulse response function $(h(t))$ which when convolved with $x(t)$ best fits $y(t)$ in the least squares sense. By mathematical definition, the estimated $h(t)$ represents the ABP response to a single, solitary cardiac contraction (normalized approximately by the average $\mathrm{PP}$ ). The impulse response function is specifically estimated according to the following autoregressive exogenous input equation:

$$
y(t)=\sum_{k=1}^{m} a_{k} y(t-k)+\sum_{k=1}^{n} b_{k} x(t-k)+e(t)
$$

where $e(t)$ is the unmeasured residual error, $\left\{a_{k}, b_{k}\right\}$ are unknown parameters, and $m$ and $n$ limit the number of these parameters (model order) [23]. For a fixed model order, the parameters are estimated from $x(t)$ and $y(t)$ in closed-form through the least-squares minimization of the residual error (i.e., linear least squares estimation) [23]. The model order is determined by minimizing the minimum description length criterion, which penalizes for unnecessary parameters [23]. With the estimated parameters $\left\{\hat{a}_{k}, \hat{b}_{k}\right\}, h(t)$ is computed as follows:

$$
h(t)=\sum_{k=1}^{m} \hat{a}_{k} h(t-k)+\sum_{k=1}^{n} \hat{b}_{k} \delta(t-k)
$$

where $\delta(t)$ is the unit impulse function.

Next, the Windkessel time constant $\tau$ is determined over the interval of $h(t)$ ranging from 2 to $4 \mathrm{~s}$ after the time of its maximum value based on the following exponential equation:

$$
h(t)=A e^{-\frac{t}{\tau}}+w(t) .
$$

The parameters $A$ and $\tau$ are estimated through the least squares minimization of the unmeasured residual error $w(t)$. This opti- 
mization problem is solved via linear least squares estimation by first log transforming $h(t)$. In theory, accurate determination of the Windkessel time constant $\tau$ is achieved by virtue of $h(t)$ coupling the long time scale variations in $x(t)$ to $y(t)$.

Finally, $\mathrm{CO}$ is computed to within a constant scale factor equal to $1 / \mathrm{AC}$ via Ohm's law

$$
\mathrm{CO} \propto \frac{\frac{1}{N} \sum_{t=1}^{N} y(t)}{\tau}
$$

Here, $N$ represents the number of samples in the digitized ABP waveform segment.

\section{METHODS}

\section{A. Experimental Protocol}

Six Yorkshire swine (30-34 kg) were studied under a protocol approved by the MIT Committee on Animal Care. The animals were given intramuscular tiletamine-zolazepam, xylazine, and atropine prior to endotracheal intubation. The swine were then maintained in a deep plane of anesthesia with inhaled isoflorane $0.5 \%-4 \%$. Positive-pressure mechanical ventilation at a rate of $10-15$ breaths/min and a tidal volume of $500 \mathrm{ml}$ was employed.

Physiologic transducers were placed as follows. 7.5 French sheath introducers (Arrow International, Reading, PA) were placed in the bilateral femoral arteries. A micromanometer-tipped catheter (SPC 350, Millar Instruments, Houston, TX) was fed retrograde to the thoracic aorta from the femoral artery for central ABP. The catheter was specifically positioned to achieve a diastolic decay that appeared as exponential as possible (i.e., at an arterial location for which the confounding wave phenomena was least apparent). The second introducer was attached to stiff fluid-filled tubing (Arrow International) and an external pressure transducer (TSD104A, Biopac Systems, Santa Barbara, CA) for femoral ABP. The chest was opened with a midline sternotomy. An ultrasonic flow probe was placed around the aortic root for reference $\mathrm{CO}$ (T206 with A-series probes, Transonic Systems, Ithaca, NY). Finally, a 23- or 25-gauge angiocatheter was placed as distal as possible to the brachial artery and attached to an external pressure transducer via short, rigid tubing for "radial" ABP. Each transducer output was interfaced to a microcomputer via an analog-to-digital conversion system (MP150WSW, Biopac Systems). The data were recorded at a sampling rate of $250 \mathrm{~Hz}$ and 16-bit resolution.

In each animal, a subset of the following interventions was performed over the course of 75 to $150 \mathrm{~min}$ to vary $\mathrm{CO}$ and other hemodynamic parameters: infusions of volume, phenylephrine, dobutamine, isoproterenol, esmolol, nitroglycerine, and progressive hemorrhage. Several infusion rates were implemented followed by brief recovery periods.

\section{B. Data Analysis}

The mathematical analysis technique was applied off-line to six-minute intervals (overlapping by $3 \mathrm{~min}$ ) of the "radial" and femoral ABP waveforms re-sampled at $90 \mathrm{~Hz}$ in order to estimate proportional $\mathrm{CO}$ trends. The corresponding reference $\mathrm{CO}$ trends were established by averaging the aortic flow probe measurements over the identical time intervals.

\section{Statistical Analysis}

To compare an estimated, proportional $\mathrm{CO}$ trend with the corresponding absolute aortic flow probe trend, the former trend was first scaled to have the same mean value as the latter trend for each animal. Then, the root-mean-squared-normalized-error (RMSNE) of the calibrated CO estimates (normalized by the aortic flow probe values and given in percent) was computed as a metric for comparison. The correlation coefficient between the estimated and aortic flow probe trends was also calculated as a scale-invariant metric for comparison.

\section{RESUlTS}

Fig. 1 illustrates samples of the digitized central, "radial," and femoral ABP waveforms. The central ABP sample is from the beginning of the recording period in which the aortic catheter was positioned such that the diastolic decay appeared as a pure exponential. However, the diastolic decay of central ABP did not consistently appear as a pure exponential throughout the recording period in which various interventions were employed. The "radial" and femoral ABP samples are from the same time but later in the recording period. Although the diastolic decay of the femoral ABP sample appears smooth, it cannot be adequately represented by a single exponential function.

Table I summarizes the results for each animal, and Figs. 5 and 6 are examples of the corresponding trends for animal 1 (worst result) and animal 5 (best result). The table and figures illustrate the wide physiologic range imposed by the interventions and strong agreement between the estimated $\mathrm{CO}$ and aortic flow probe trends with an overall CO RMSNE of $14.6 \%$. (The femoral ABP waveform of animal 4 is not included in Table I due to excessive damping. However, we note that the technique achieved a CO RMSNE of $18.2 \%$ with respect to this waveform. See Section V for implications.) This strong agreement was confirmed by high overall correlation coefficients (mean \pm $\mathrm{SD})$ between the aortic flow probe trends and the $\mathrm{CO}$ trends estimated from the "radial" $(0.84 \pm 0.07)$ and femoral $(0.86 \pm 0.05)$ ABP waveforms. Additionally, Table II shows that the $\mathrm{CO}$ error (difference between calibrated, estimated CO trends and corresponding aortic flow probe trends) was only mildly correlated with mean ABP (MAP) and essentially uncorrelated with $\mathrm{CO}$ and heart rate (HR). Finally, we found no visual systematic dependence of the $\mathrm{CO}$ error on any specific type of intervention. (That is, for example, the overestimation of CO in Fig. 5 during phenylephrine administration was an isolated event.)

\section{DISCUSSION}

We have developed a novel mathematical analysis technique for continuously monitoring changes in $\mathrm{CO}$ from a single peripheral ABP waveform. The technique analyzes 6-min intervals of a peripheral ABP waveform so as to establish the pure exponential decay that would eventually result if pulsatile activity abruptly ceased. More specifically, the technique estimates the 
TABLE I

SUMMARY OF RESULTS

\begin{tabular}{cccccc}
\hline ANIMAL & CO RANGE & MAP RANGE & HR RANGE & \multicolumn{2}{c}{ CO RMSNE [\%] } \\
[L/MIN] & {$[$ [MMHG] } & {$[$ [BPM] } & FEMORAL & "RADIAL" \\
\hline 1 & $1.6-5.2$ & $29-100$ & $96-180$ & 19.9 & 19.1 \\
2 & $2.3-4.2$ & $54-127$ & $101-204$ & 10.2 & 16.0 \\
3 & $1.9-5.8$ & $70-120$ & $96-186$ & 8.8 & 16.7 \\
4 & $1.3-4.3$ & $27-106$ & $103-198$ &.-- & 12.3 \\
5 & $2.4-5.0$ & $65-118$ & $91-198$ & 10.2 & 8.0 \\
6 & $2.3-5.6$ & $52-108$ & $109-177$ & 17.1 & 14.7 \\
& & & & & \\
TOTAL & $1.3-5.8$ & $27-127$ & $91-204$ & 14.0 & 15.0 \\
\hline
\end{tabular}

* The technique of Fig. 4 was applied to femoral and "radial" ABP waveforms in six open-chest swine and achieved an overall $\mathrm{CO}$ error of $14.6 \%$ with respect to the aortic flow probe over a wide physiologic range. (The femoral $\mathrm{ABP}$ waveform of animal 4 is not included due to excessive damping. However, note that the technique achieved a $\mathrm{CO}$ error of $18.2 \%$ with respect to this waveform.).
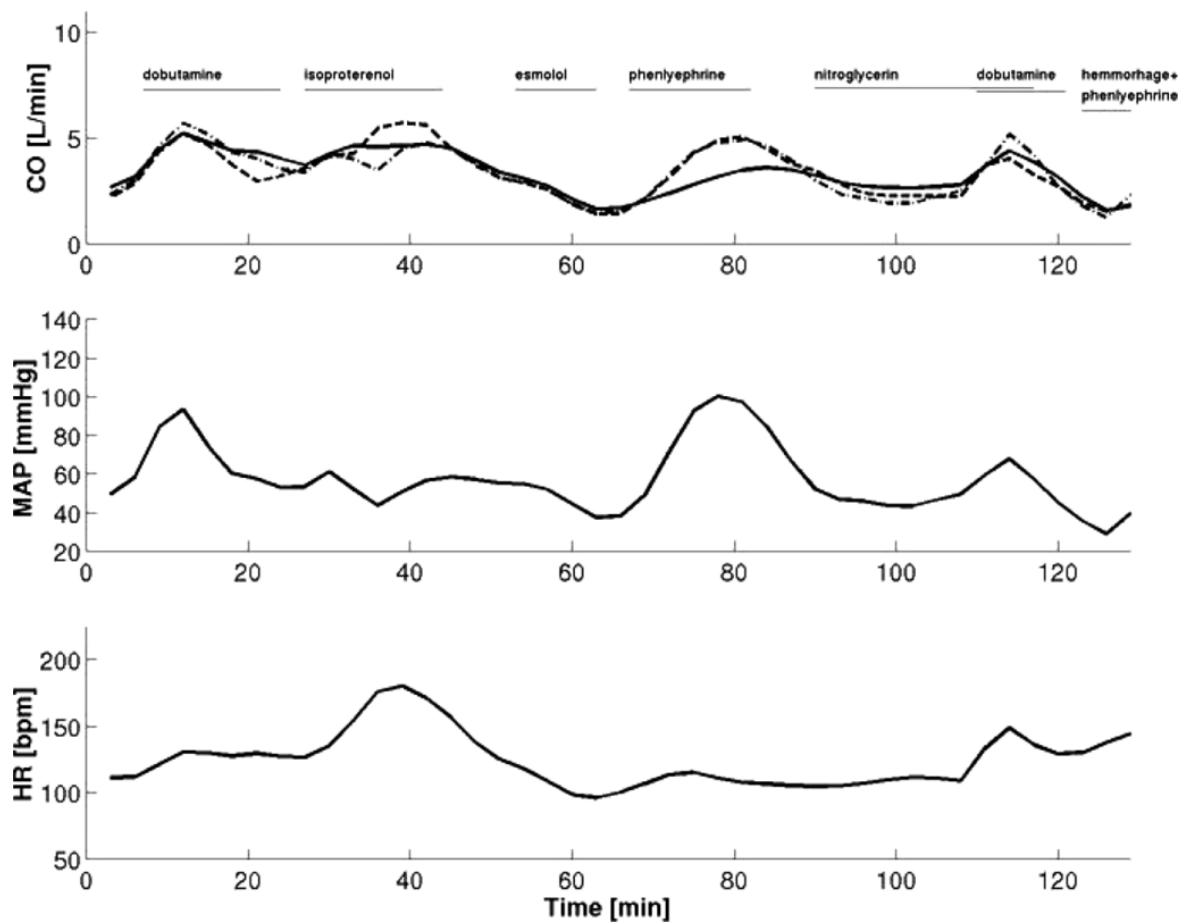

Fig. 5. Trend results for animal 1 in Table I (worst result). The top panel illustrates the aortic flow probe CO trend (solid), estimated and calibrated CO trends from the "radial" (dashed) and femoral (dashed-dotted) ABP waveforms, and the intervention and duration (underline). The bottom two panels illustrate the mean ABP (MAP) and HR trends.

ABP response to a single, solitary cardiac contraction (i.e., impulse response $h(t)$ in Fig. 4). Then, the Windkessel time constant $\tau$, which is equal to the product of the TPR and nearly constant true total AC (see later), is measured by fitting an exponential to the tail end of this estimated response once the faster wave reflections have vanished (Fig. 4). Finally, proportional $\mathrm{CO}$ is computed via Ohm's law. We tested the technique in acute swine experiments, and our results showed strong agreement with aortic flow probe measurements over a wide physiologic range with an overall $\mathrm{CO}$ error of $14.6 \%$.

To the best of our knowledge, the technique introduced herein represents the very first attempt to extract $\mathrm{CO}$ information from ABP variations occurring over time scales greater than a cardiac cycle (i.e., beat-to-beat variability). Thus, in principle, the performance of our technique should be less dependent on the ABP measurement site than previous techniques [as beat-to-beat ABP variations are consistent throughout the arterial tree (Fig. 3)]. Furthermore, our technique does not attempt to model phys- ically the highly complex wave effects. Rather, the technique lets the measured waveform determine the wave characteristics of a particular individual over a specific time interval through estimation of an individualized impulse response of arterial dynamics (see later). Thus, our technique should be relatively unaffected by differences in wave phenomena between subjects and disease states. Finally, since our technique focuses the analysis on low frequency behavior rather than high frequency detail, the technique should not be as reliant as previous techniques on ABP transducers with excellent frequency response characteristics (e.g., micromanometer-tipped catheters) and may even perform adequately with damped fluid-filled catheter systems (Table I caption).

The idea here of analyzing beat-to-beat ABP variability in order to circumvent confounding wave phenomena is supported by a previous technique introduced by Quick et al. [24]. These investigators extended the standard Windkessel theory (Fig. 1) to account for distributed effects by introducing frequency 

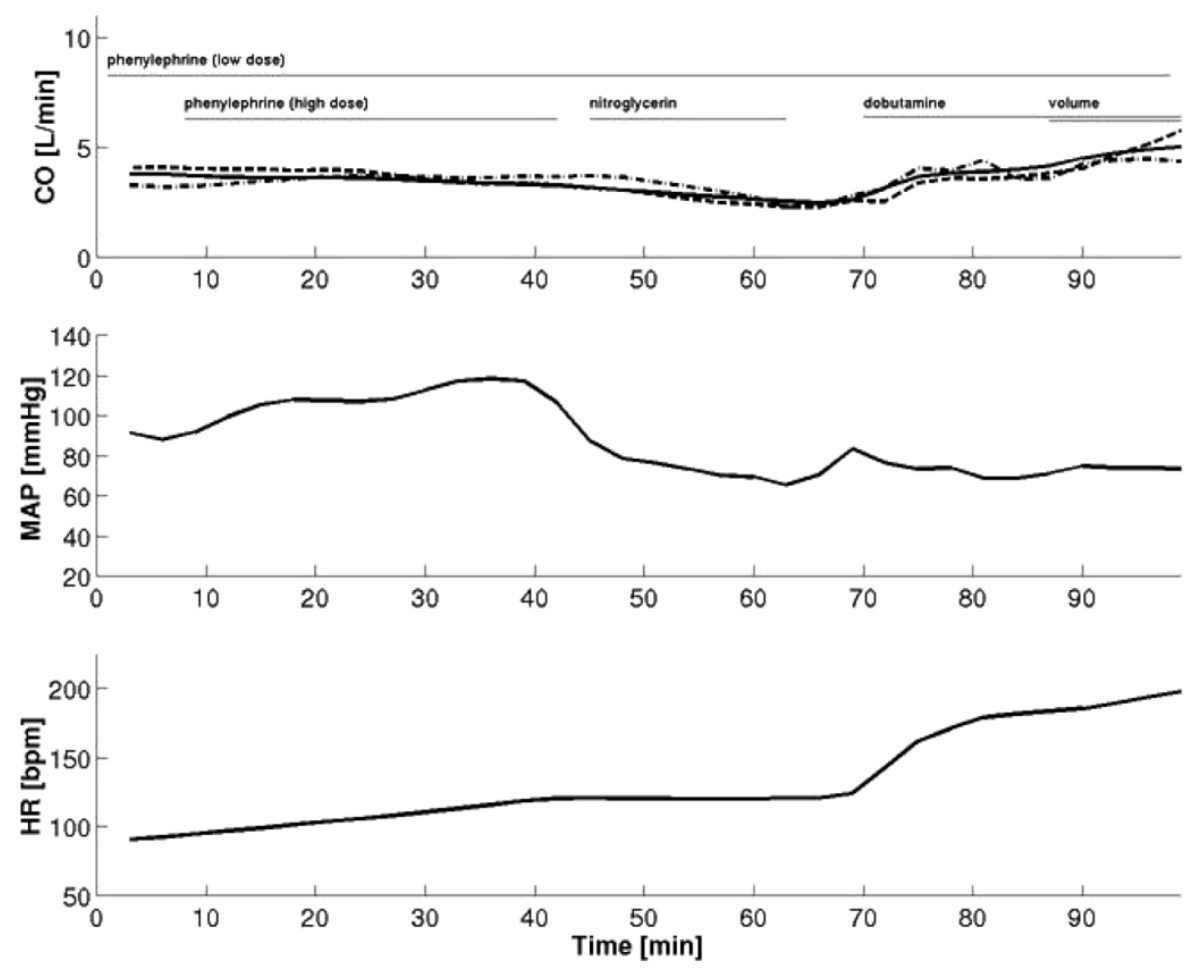

Fig. 6. Trend results for animal 5 in Table I (best result). See Fig. 5 caption.

dependence in the $\mathrm{AC}$ element. They referred to this frequency dependent transfer function as the apparent AC. They then developed a technique to estimate the apparent $\mathrm{AC}$ based on the arterial input impedance and TPR as determined from measurements of aortic pressure and flow. They also argued, much like we have, that the confounding effects of wave reflections disappear at frequencies below the mean HR and the apparent $\mathrm{AC}$ becomes the true total AC. (Note that their definition of true total $\mathrm{AC}$ is equivalent to what we refer to here as simply AC.) Thus, based on this argument and their estimate of the apparent $\mathrm{AC}$, they have introduced a technique for determining AC that specifically utilizes the information present in the beat-to-beat variations in aortic pressure and flow. In contrast to the technique of Quick et al., our technique aims to determine proportional $\mathrm{CO}$ from a peripheral $\mathrm{ABP}$ waveform. While our technique may also provide a less invasive means for determining $\mathrm{AC}$ with an additional thermodilution measurement $((\mathrm{CO} \cdot \tau) / \mathrm{MAP})$, for reasons described later, it is not able to determine the entire apparent $\mathrm{AC}$ transfer function.

Our technique is also related to previous quantitative investigations, which have utilized the impulse response to characterize arterial tree dynamics (e.g., [25]). In these investigations, the impulse response was determined via highly invasive means by either experimental application of an impulse of flow (via an occluder around the ascending aorta) or estimation from measurements of aortic pressure and flow. While our technique is advantageous in that it was designed to be minimally invasive, we note that it does not aim to estimate the entire arterial tree impulse response (or equivalently, the entire arterial input impedance). To identify this impulse response, a system identification problem must be solved in which aortic flow is the input and ABP is the output. Since our technique aims to determine proportional $\mathrm{CO}$, it constructs a first-order approximation of the aortic flow signal based on the available peripheral ABP waveform using an impulse ejection model. While this approximation is clearly not able to represent the actual finite ejection time of aortic flow, it has relatively little effect in terms of estimating the Windkessel time constant $\tau$. More specifically, as a consequence of this approximation, the estimated impulse response actually represents the convolution of the arterial tree impulse response with what may be thought of as a single averaged beat of the phasic aortic flow. Importantly, however, this filtering by the phasic aortic flow does not preclude the subsequent determination of $\tau$ from our impulse response estimate, because the ejection interval of aortic flow is usually much smaller than $\tau$.

Our impulse response estimate also differs from the arterial tree impulse response, as measured via an actual impulse of flow, in that the latter impulse response may ultimately decay to the critical closing pressure [13]. However, we note that the absence of the critical closing pressure in our impulse response estimate usually does not preclude reliable, subsequent determination of the Windkessel time constant $\tau$. More specifically, our technique estimates the impulse response from the information present in the measured $\mathrm{ABP}$ waveform. Thus, if the measured $\mathrm{ABP}$ is above the critical closing pressure, then the technique cannot "see" this pressure. In this way, the technique is effectively able to determine the pure exponential decay that would occur in an arterial system without a critical closing pressure. However, if $\mathrm{ABP}$ is below this pressure, then the resulting $\tau$ estimate may suffer (see later for further discussion of the hypotension case).

In principle, $\tau$ cannot be determined by simply fitting single exponential functions directly to the diastolic intervals of peripheral ABP waveforms, because these intervals usually do not appear as pure exponential decays [Fig. 2(b)]. One may therefore 
be tempted to measure $\tau$ through a multiple exponential fit. However, the energy contribution of the confounding wave reflections is relatively large over time scales within a diastolic interval, and the fit may therefore favor smaller time constants. That is, the signal-to-noise (SNR) conditions over these short time scales are not well suited for measuring $\tau$. One may consider improving the SNR conditions by low-pass filtering (smoothing) the diastolic decay intervals. However, the filter bandwidth (degree of smoothing) would be difficult to specify without prior knowledge of the value of $\tau$, which is the very parameter sought for measurement (i.e., chicken and egg problem).

To buttress the above theoretical arguments, we sought to monitor CO from our "radial" ABP waveforms by estimating $\tau$ from only the intrabeat ABP information as follows. First, we fitted a single exponential function and a fixed and optimal number of multiple, complex exponential functions directly to the downstroke (i.e., from peak to minimum) as well as to the diastolic interval (as precisely determined with the available aortic flow waveform) of each ABP pulse. Then, for the multiple exponential fits, we determined $\tau$ for each ABP pulse by extrapolating the estimated exponential functions toward low-pressure values and fitting a mono-exponential function to the extrapolated values. Finally, we established the average $\tau$ over six-minute intervals of the ABP waveform by computing the arithmetic mean and the median (robust to outliers) of $\tau$ estimated from each beat. The best result we were able to achieve with this intrabeat approach was an overall RMSNE of $22.8 \%$ (i.e., a $52 \%$ increase in CO error with respect to our technique). Moreover, in contrast to our technique (Table II), the CO error of this approach was dependent on the HR, as this parameter established the number of data samples available to the fitting procedure.

We believe that the above comparative study demonstrates that important $\mathrm{CO}$ information is indeed present in beat-to-beat ABP variations. However, we note that further studies comparing our technique with the intrabeat techniques of Wesseling et al. [20] and Linton et al. [21] should also be conducted. We feel that such future studies will only be meaningful provided that the techniques are compared with respect to the same data set.

Unlike the above intrabeat techniques, our technique, in its present form, is unable to provide beat-to-beat $\mathrm{CO}$ estimation. Rather, our technique analyzes the interbeat $A B P$ variations with the aim of obtaining an improved estimate of average $\mathrm{CO}$. We feel that attempts to improve the accuracy of the $\mathrm{CO}$ estimation, even at the cost of temporal resolution, are worthwhile from a clinical point of view. That is, although most previous techniques offer beat-to-beat $\mathrm{CO}$, they have still not been widely adopted presumably due to accuracy concerns.

We specifically chose to analyze six-minute intervals of a peripheral ABP waveform, because we felt, based on our background in HR variability analysis, that there would be enoughlong time scale or beat-to-beat variability to determine the Windkessel time constant accurately. Moreover, automatic estimation of $\mathrm{CO}$ at six-minute intervals would represent a significant improvement upon discrete, operator-required determinations of $\mathrm{CO}$ by the clinical thermodilution method (assuming similar accuracy). Nevertheless, enhancement of the temporal resolution of the technique without significant loss of accuracy is desirable. Preliminary studies show that six-minute intervals may be reduced to,
TABLE II

CORRELATION COEFFICIENT MATRIX

\begin{tabular}{lccc}
\hline & $\mathrm{CO}$ & $\mathrm{MAP}$ & $\mathrm{HR}$ \\
\hline "RADIAL" ABP CO ERROR & 0.11 & 0.47 & -0.08 \\
FEMORAL ABP CO ERROR & 0.09 & 0.32 & 0.14 \\
\hline * The CO error indicated in Table I was only mildly corre- \\
lated with the mean ABP or MAP and essentially uncor- \\
related with CO and HR.
\end{tabular}

for example, 90-second or three-minute intervals without materially affecting our results (i.e., an increase in $\mathrm{CO}$ error of $<2 \%$ ). More detailed studies are required to determine the optimal interval length for analysis.

On the other hand, we studied an acute swine model in which the beat-to-beat variability was depressed due to the inhibitive effects of anesthesia on autonomic function. Even under these circumstances, the technique was able to predict $\mathrm{CO}$ changes accurately (Table I). These results suggest that the technique may perform reliably in individuals with depressed beat-to-beat variability due to age or disease. However, we suspect that our technique may perform even more accurately when applied to individuals with normal or enhanced autonomic states. Further experimental studies are necessary to determine the extent to which beat-to-beat variability affects the performance of our technique.

A potential source of error in our technique is that the swine AC may not be perfectly constant over the wide pressure range considered here (i.e., nonlinear AC). Previous investigators have shown differential AC to decrease with increasing MAP in several different species (e.g., [10], [26], and [27]). If this pattern applied to our swine model, then we would expect our technique to overestimate grossly $\mathrm{CO}$ at high MAP states and underestimate $\mathrm{CO}$ at low MAP states (i.e., strong, positive correlation between $\mathrm{CO}$ error and MAP). Although the correlation between $\mathrm{CO}$ error and MAP is positive, the degree of the correlation is mild (Table II). This result suggests that the AC in our swine model may be nearly linear over a wide pressure range, which is consistent with the canine studies of Bourgeois et al. [8], [9] and a human study in which differential compliance was measured in the excised aorta at autopsy [10]. Previous studies reporting more significant degrees of nonlinearity may be a consequence of errors associated with the estimation of AC.

In a related vein, our technique assumes that the viscous component of the arterial wall is negligible [13]. Viscoelastic dynamics of the arterial wall could potentially obscure to some degree the manifestation of the Windkessel time constant $\tau$ in our estimated impulse response and therefore also be a source of error. On the other hand, we note that the time constants governing viscoelasticity may usually be fast enough [28] such that they effectively die out by the time the exponential function is fit to the estimated impulse response (Fig. 4).

Another possible source of error in our technique is that the contribution of peripheral venous pressure (PVP) to the pressure gradient across the resistance beds is assumed to be negligible. For example, the technique determines proportional $\mathrm{CO}$ by dividing mean $\mathrm{ABP}$ (MAP) rather than the difference between MAP and mean PVP, with the Windkessel time constant. Under most circumstances, PVP is much smaller than ABP. However, under extreme hypotensive conditions for example, this may no 
longer be the case, and our technique may therefore overestimate the pressure gradient across the resistance beds. Additionally, as mentioned above, the estimated Windkessel time constant may also be in error due to omission of the critical closing pressure. Since we only found mild correlation between CO error and MAP (Table II), either the ABP in our animal model was always sufficiently large with respect to PVP (as well as the critical closing pressure) or there were some fortuitous cancellation of errors. Regardless, during frank hypotension, the precise value of $\mathrm{CO}$ may not be clinically important.

The new technique that we presented and validated here mathematically analyzes a single peripheral ABP waveform over long time scales in order to measure $\mathrm{CO}$ to within a constant scale factor. The technique may therefore be utilized to monitor quantitatively changes in $\mathrm{CO}$. The proportional $\mathrm{CO}$ may be calibrated, if desired, with a single, absolute $\mathrm{CO}$ measurement (e.g., thermodilution). For normal individuals, it may be possible to determine the proportionality constant from a nomogram. However, we believe determination of the proportionality constant is unnecessary in the context of continuous monitoring in the acute setting in which only $\mathrm{CO}$ changes are clinically relevant. In the future, we plan to conduct human testing of the technique in which success will obviously be dependent on the degree of linearity of the human AC. With such successful future testing, the technique may potentially be employed in intensive care units and surgical suites in which invasive radial ABP waveforms are routinely measured or in primary care settings, emergency rooms, and regular hospital beds in which noninvasive measurements of ABP waveforms (e.g., arterial tonometry) could easily be obtained. Finally, the technique is general and may be applied to ABP waveforms measured at any site in the systemic or pulmonary arterial tree.

\section{REFERENCES}

[1] K. C. Ehlers, K. C. Mylrea, C. K. Waterson, and J. M. Calkins, "Cardiac output measurements. A review of current techniques and research," Ann. Biomed. Eng., vol. 14, no. 3, pp. 219-239, 1986.

[2] A. F. Connors, T. Speroff, N. V. Dawson, C. Thomas, F. E. Harrell, D. Wagner, N. Desbiens, L. Goldman, A. W. Wu, R. M. Califf, W. J. Fulkerson, H. Vidaillet, S. Broste, P. Bellamy, J. Lynn, and W. A. Knaus, "The effectiveness of right heart catheterization in the initial care of critically ill patients," JAMA, vol. 276, no. 11, pp. 889-897, 1996.

[3] T. Kenner, "ABP and its measurement," Basic Res. Cardiol., vol. 83, no. 2, pp. 107-121, 1988.

[4] B. P. M. Imholz, W. Wieling, G. A. van Montfrans, and K. H. Wesseling, "Fifteen years experience with finger arterial pressure monitoring: assessment of the technology," Cardiovasc. Res., vol. 38, pp. 605-616, 1998.

[5] J. Erlanger and D. R. Hooker, "An experimental study of blood-pressure and of pulse-pressure in man," Bull. Johns Hopkins Hosp., vol. 12, pp. 145-378, 1904

[6] C. F. Starmer, P. A. McHale, F. R. Cobb, and J. C. Greenfield, "Evaluation of several methods for computing stroke volume from central aortic pressure," Circ. Res., vol. 33, pp. 139-148, 1973.

[7] P. D. Verdouw, J. Beaune, J. Roelandt, and P. G. Hugenholtz, "Stroke volume from central aortic pressure? A critical assessment of the various formulae as to their clinical value," Basic Res. Cardiol., vol. 70, pp. $377-389,1975$

[8] M. J. Bourgeois, B. K. Gilbert, G. von Bernuth, and E. H. Wood, "Continuous determination of beat-to-beat stroke volume from aortic pressure pulses in the dog," Circ. Res., vol. 39, no. 1, pp. 15-24, 1976.

[9] M. J. Bourgeois, B. K. Gilbert, D. E. Donald, and E. H. Wood, "Characteristics of aortic diastolic pressure decay with application to the continuous monitoring of changes in peripheral resistance," Circ. Res., vol. 35 , pp. 56-66, 1974.
[10] P. Hallock and J. C. Benson, "Studies on the elastic properties of human isolated aorta," Am. J. Physiol., vol. 16, pp. 595-602, 1937.

[11] J. W. Remington and W. F. Hamilton, "The construction of a theoretical cardiac ejection curve from the contour of the aortic pressure pulse," $\mathrm{Am}$. J. Physiol., vol. 144, pp. 546-556, 1945.

[12] M. F. O'Rourke and T. Yaginuma, "Wave reflections and the arterial pulse," Arch. Intern. Med., vol. 144, pp. 366-371, 1984.

[13] A. Noordergraaf, Circulatory System Dynamics. New York: Academic Press, 1978.

[14] R. M. Cundick and R. M. Gardner, "Clinical comparison of pressurepulse and indicator-dilution cardiac output determination," Circulation, vol. 62, no. 2, pp. 371-376, 1980.

[15] J. D. Redling and M. Akay, "Noninvasive cardiac output estimation: a preliminary study," Biol. Cybern., vol. 77, pp. 111-122, 1997.

[16] W. Welkowitz, Q. Cui, Y. Qi, and J. B. Kostis, "Noninvasive estimation of cardiac output," IEEE Trans. Biomed. Eng., vol. 38, no. 11, pp. 1100-1105, Nov. 1991.

[17] C. Cerutti, M. P. Gustin, P. Molino, and C. Z. Paultre, "Beat-to-beat stroke volume estimation from aortic pressure waveform in conscious rats: comparison of models," Am. J. Physiol., vol. 281, pp. H1148-H1155, 2001.

[18] I. Gratz, J. Kraidin, A. G. Jacobi, N. G. deCastro, P. Spagna, and G. E. Larijani, "Continuous noninvasive cardiac output as estimated from the pulse contour curve," J. Clin. Monit., vol. 8, pp. 20-27, 1992.

[19] J. F. Martin, L. B. Volfson, V. V. Kirzon-Zolin, and V. G. Schukin, "Application of pattern recognition and image classification techniques to determine continuous cardiac output from the arterial pressure waveform,” IEEE Trans. Biomed. Eng., vol. 41, no. 10, pp. 913-920, Oct. 1994.

[20] K. H. Wesseling, J. R. C. Jansen, J. J. Settels, and J. J. Schreuder, "Computation of aortic flow from pressure in humans using a nonlinear, threeelement model," Am. J. Physiol., vol. 74, no. 5, pp. 2566-2573, 1993.

[21] N. W. F. Linton and R. A. F. Linton, "Estimation of changes in cardiac output from the abp waveform in the upper limb," Br. J. Anaesth., vol. 86, pp. 486-496, 2001.

[22] M. L. Appel, R. D. Berger, J. P. Saul, J. M. Smith, and R. J. Cohen, "Beat to beat variability in cardiovascular variables: noise or music?," JACC, vol. 14, no. 5, pp. 1139-1148, 1989.

[23] L. Ljung, System Identification: Theory for the User. Englewood Cliffs, NJ: Prentice-Hall, 1987.

[24] C. M. Quick, D. S. Berger, D. A. Hettrick, and A. Noordergraaf, "True arterial system compliance estimated from apparent arterial compliance," Ann. Biomed. Eng., vol. 28, pp. 291-301, 2000.

[25] P. Sipkema, N. Westerhof, and O. S. Randall, "The arterial system characterized in the time domain," Cardiovasc. Res., vol. 14, no. 5, pp. 270-279, 1980.

[26] J. K. Li and Y. Zhu, "Arterial compliance and its pressure dependence in hypertension and vasodilation," Angiology, vol. 45, no. 2, pp. 113-117, 1994.

[27] G. J. Langewouters, K. H. Wesseling, and W. J. A. Goedhard, "The static elastic properties of 45 human thoracic and 20 abdominal aortas in vitro and the parameters of a new model," J. Biomech., vol. 17, no. 6, pp. 425-435, 1984

[28] K. H. Wesseling, H. Weber, and B. de Wit, "Estimated five component viscoelastic model parameters for human arterial walls," J. Biomech., vol. 6, no. 1, pp. 13-24, 1973.

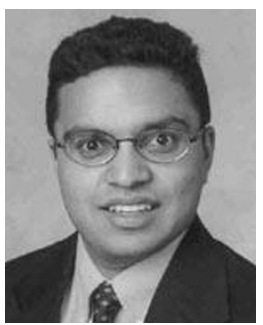

Ramakrishna Mukkamala (M'02) received the B.S.E. degree in biomedical/electrical engineering from Duke University, Durham, NC, in 1993 and the S.M. and Ph.D. degrees in electrical engineering and computer science from the Massachusetts Institute of Technology, Cambridge, in 1995 and 2000, respectively.

He was a Postdoctoral Fellow/Research Engineer in the Harvard-MIT Division of Health Sciences and Technology from 2000 to 2002. Since then, he has been on the faculty of Electrical and Computer Engineering at Michigan State University, East Lansing, where he is currently an Assistant Professor. His research interests include biomedical signal processing and identification, modeling of physiologic systems, and cardiovascular physiology. 


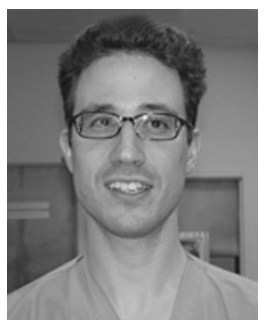

Andrew T. Reisner received the B.S. degree in mechanical engineering and biological sciences at Stanford University, Stanford, CA, in 1992, the M.D. degree from Harvard Medical School, Cambridge, MA, in 1997, and trained in emergency medicine at the Harvard-affiliated Emergency Medicine Residency program.

$\mathrm{He}$ is presently an Attending Physician at Massachusetts General Hospital in the Department of Emergency Medicine and a visiting scientist at the the Massachusetts Institute of Technology, Cambridge. His research involves the intersection of diagnostic expert systems, medical sensor technology, and the clinical problem of circulatory shock.

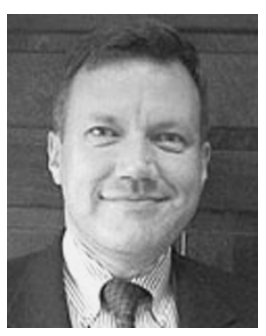

Horacio M. Hojman received the M.D. degree in 1984 from the Universidad del Salvador, Buenos Aires, Argentina.

He went on to complete a residency in General Surgery and a fellowship in Cardiovascular Surgery in Argentina before coming to the United States in 1992. He underwent further training in General Surgery in Brooklyn, NY and Surgical Critical Care at the Massachusetts General Hospital, Boston. Currently, he is an Associate Professor of Surgery in the section of Trauma and Surgical Critical Care, Yale University School of Medicine, New Haven, CT. His research involves wound ballistics and minimally invasive monitoring in trauma and shock.

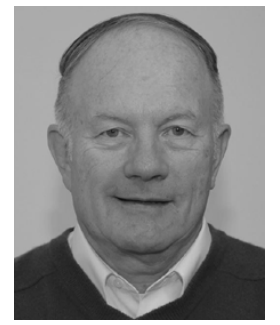

Roger G. Mark (S'59-M'83-SM'95) is the Distinguished Professor of Health Sciences and Technology and Professor of Electrical Engineering at the Massachusetts Institute of Technology, Cambridge. He remains active in the practice of primary care internal medicine with a focus on geriatrics, and is Senior Physician at the Beth Israel Deaconess Medical Center. His current research interests are physiological signal processing, cardiovascular modeling, and intelligent patient monitoring.

Dr. Mark is a fellow of the American College of Cardiology, a founding fellow of the American Institute of Medical and Biological Engineering, and past president and board member of Computers in Cardiology, Inc.

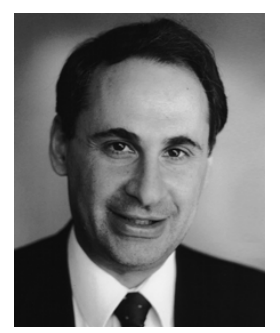

Richard J. Cohen is Whitaker Professor in Biomedical Engineering in the Harvard University-Massachusetts Institute of Technology Division of Health Sciences and Technology, Cambridge. His research interests involve the application of physics and engineering to the development of new diagnostic and therapeutic cardiovascular technologies in the areas of cardiac electrophysiology, hemodynamic regulation, and ultrasound imaging. A particular focus of his work involves the stochastic analysis of second-to-second fluctuations in physiologic signals in order to develop new diagnostic tools such as microvolt T-wave alternans testing to assess susceptibility to ventricular dysrhythmias, and cardiovascular system identification to assess closed-loop hemodynamic regulation. 